\title{
Robust Vehicle Infrastructure Cooperative Localization in Presence of Clutter
}

\author{
Dhiraj Gulati* $^{\dagger}$, Vincent Aravantinos*, Nikhil Somani ${ }^{\dagger}$ and Alois Knoll ${ }^{\dagger}$ \\ *fortiss GmbH, München, Germany. \{gulati, aravantinos\}@fortiss.org \\ †Technische Universität München, Garching bei München, Germany.dhiraj.gulati@tum.de, \{knoll, somani\}@in.tum.de
}

\begin{abstract}
One of the primary challenges for a successful Highly Assisted and/or Autonomous Vehicle is its localization. To improve the precision of location of the vehicle, not only the internal sensors are being used, but also using data from external sensors is attracting increasing attention from the research community. One such proposed sensor is an infrastructure RADAR which can be used to improve the localization of the egovehicle. Although a RADAR indeed is a supplementary source of information, it suffers a unique type of clutter which have trajectories like real objects and can therefore result in "ghost measurements", i.e., measurements which do not correspond to any real vehicles. This deteriorates the quality of the fused state estimates. This paper proposes a robust method to fuse the RADAR readings in presence of such outliers. This methodology builds upon a previously proposed solution where the problem was formulated as a factor graph. The RADAR measurements were added as a novel constraint of sum of inter-vehicle distance, called Topology Factor. Our previous work assumed clutter free environment. This paper proposes a novel robust Topology Factor which is also resilient against above mentioned outliers. Simulations (based on real data) show promising results in the direction of lowering the degradation of fused state estimates in presence of such clutter.
\end{abstract}

\section{INTRODUCTION}

The ability to communicate in real time using technologies like $5 G$ [1] accompanied with increasing deployment of sensors in the environment has enabled cooperative localization to become a viable solution for Highly Automated and fully Autonomous vehicles.

Cooperative localization is not a new concept. One of the earliest work of Kurazume et. al. [2] showed the potential and power of the concept in the domain of Autonomous Robots. Since then researchers have proposed many innovative solution using various Data Fusion algorithms and technologies. Various solutions use Kalman Filter (or its non-linear extensions like Extended Kalman Filter or Unscented Kalman Filter) [3], [4] to track the cooperative state variables and the corresponding covariances. Howard et. el. [5] uses Maximum Likelihood Estimation (MLE) to achieve the cooperative localization by combining relative measurements between robots in a least square formulation. This formulation is solved for the fused state estimates of the robots. Ahmad et. el. [6] does the same but also includes moving landmarks. Various other novel works include, but not limited to, Markov Localization [7], split covariance intersection filter [8], random finite set framework [9], Symmetric Measurement Equation Filter [10] and non-parametric Belief Propagation [11].
Graph-based methods have also been used to formulate and solve the problem of cooperative localization. GTSAM [12] and $\mathrm{g}^{2} \mathrm{o}$ [13] are two such graph-based frameworks which facilitate the state estimation for robots. Our previous work [14] used the GTSAM framework to propose an innovative solution of Topology Factor formulated from the external radar measurements to perform cooperative localization. In addition other challenges, namely bandwidth limitations; data association uncertainties; unknown coordinate transformations; and scalability are also simultaneously addressed. [15] proposes the use of Symmetric Measurement Equation Factors to perform the cooperative localization.

Although most of the clutter handling is achieved at hardware in radar [16], some can still be present as measurements. Tracking Algorithms are able to handle it fairly well. Random Finite Set Statistics filters like GMPHD [17] and GLMB [18] drop measurements which do not meet a certain frequency threshold. Multiple-Hypothesis Tracker constructs multiple possible trees and prunes them regularly to eliminate false positives [19]. Data Association algorithms like Probability Data Association (PDA) Filter and Joint Probability Data Association (JPDA) Algorithm are able to partially avoid clutter measurements which are outside the defined Gate [20].

Graph-based methods mostly rely on the front end or any other association algorithm to handle the outliers. Our previous solution [21] used PDA Filter along side the Topology Factor solution to tackle the challenge of clutter measurements. The solution tracked the resulting Topology Measurements and applied PDA Filter to reject any measurements which did not meet the threshold and the remaining were assigned a probability. These probabilities were used as weights for the Topology Factors by the optimizer. In terms of Factor Graphs (for an overview of Factor Graphs, the reader can refer the Section IV), the proposed solution can be formulated as:

$$
f^{T}\left(X_{1}, \cdots, X_{n}\right) \triangleq \beta_{j} \cdot \delta\left(\left(\mathrm{h}\left(x_{1}, \cdots, x_{n}\right)-\left(z_{j}^{T}\right)\right)\right.
$$

where $f^{T}$ is the Topology Factor between the states $X_{i}$, $\forall i=1 \cdots n, \mathrm{~h}$ is the Topology function, $z_{j}^{T}$ is the $j^{t h}$ Topology Measurement constructed from radar, $\delta$ is the minimization function, and $\beta_{j}$ is the probability for $j^{\text {th }}$ Topology measurement obtained using PDA Filter.

On the other hand, Sünderhauf et al. [22] developed the solution of Switchable Constraints to strengthen the pose graph based solution specifically against false loop closures. They demonstrated their solution using GTSAM and $\mathrm{g}^{2} \mathrm{o}$. They do 
not use any data association algorithm in parallel to SLAM, but let the optimizer reject any false loop closures.

Therefore, the challenges are two-fold:

- Solve the problem of clutter using only the optimizer and remain independent of any other methods.

- Previous work [21] demonstrated the results using 2 vehicles simulation. The PDA Filter uses a Kalman Filter and tracks the Topology Measurements, this implies we require a Model for the Topology Measurements. For 2 vehicles we assumed a Linear Model, but for multivehicle scenario this assumption is highly inaccurate and unlikely. Such model even if it exists, is mathematically complex to arrive at. Hence the problem of multi-vehicle scenario remains unaddressed.

This work presents a new robust Topology Factor addressing the above two criterion. The simulation is run for more than two vehicles and its effectiveness is analysed using Root Mean Square Error (RMSE). The results demonstrate the feasibility of the proposed solution to keep the error in check. The simulation data is based on analysis of real radar data observed on a highway. We also compare its performance against the solution of Switchable Constraints used for Topology Factor.

The rest of the paper is structured as follows: Sec. II briefly describes the assumptions and complete scenario for cooperative localization. Sec. III and IV present an overview of radar and Factor Graphs respectively. Sec. V and VI present the proposed solution and simulation results. Finally, Sec. VII concludes the paper.

\section{Problem Description}

For sake of completeness, we describe a simple cooperative localization scenario in presence of clutter. The basic problem definition remains same as in [21] and can be seen in the Fig. 1. Dotted blue lines represent the radar coordinate system. The radar measurements are represented with stars. One extra black star represents the clutter measurement.

The assumptions are as follows:

1) Vehicles have Odometer sensors to measure the relative distance travelled in unit time.

2) Vehicles have GPS sensors to measure their position in an absolute reference of a 2D global coordinate system.

3) The infrastructure radar sensor measures positions of the vehicles in its own local 2D coordinate system. Its configuration information is not available, such that its location and orientation is unknown.

4) The vehicles and the radar Sensor can communicate in either direction to exchange data. There is no timing delay or data error in communication.

5) No mechanism is available, including communication mechanism and/or the protocol to identify individual vehicles. This introduces a challenge from the perspective of data association.

6) The radar detects clutter and can also have miss detections.

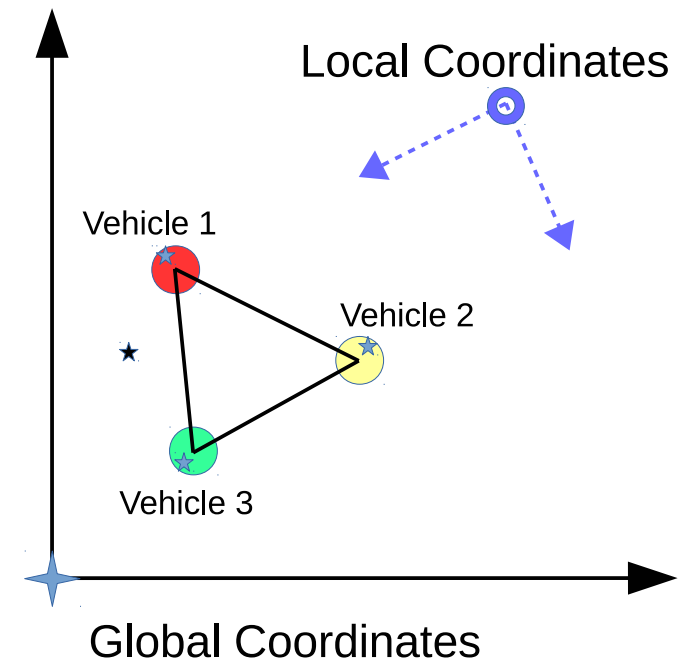

Fig. 1. Topology for vehicle infrastructure cooperative localization. Dashed Axis in blue represent the coordinate system of the radar. Stars represent the radar measurements. The black star is a clutter measurement.

Then the task of cooperative localization is to lower the error in position estimation in presence of clutter measurements by fusing the measurements from all the sensors.

\section{Clutter From Infrastructure RADAR}

This section gives a quick overview of the functioning of an Infrastructure radar. This helps in better understanding the kind of clutter which can be observed on a scenario like that of a highway. For detailed functioning of a radar, reader is advised to consult references like Radar Handbook by Skolnik [16] and online references like [23].

A typical radar deployed in a traffic scenario to oversee $4-8$ lanes is a Moving Target Indicator (MTI) Radar. Like the name suggests, the aim for an MTI Radar is to detect a moving target and reject the measurements from fixed or slowmoving unwanted targets like buildings, trees or rain. These radar types fall under Frequency-Modulated Continuous-Wave Radar (FMCW Radar). These are special kind of sensors which radiate continuous transmission power with change in the frequency. That is, the transmission signal is modulated in frequency. This increase or decrease of the frequency of the sent signal is measured against the change of frequency in the received signal. This helps in measuring the distance of the moving object and the relative velocity simultaneously.

The state-of-the-art MTI Radars use real time sophisticated circuits and physics principles to operate. Therefore, they also have hardware based filters to remove lots of clutter from the scenarios. Details of such filters can be found in [16]. This is also a topic of active reserach in radar domain. Despite all the advancements, sometimes high winds, rain and reflections result in detections which could not be filtered at hardware and appear as clutter measurements which do not belong to any real target. This can induce errors in the state estimation. All these clutter measurements which escaped the signal-level 


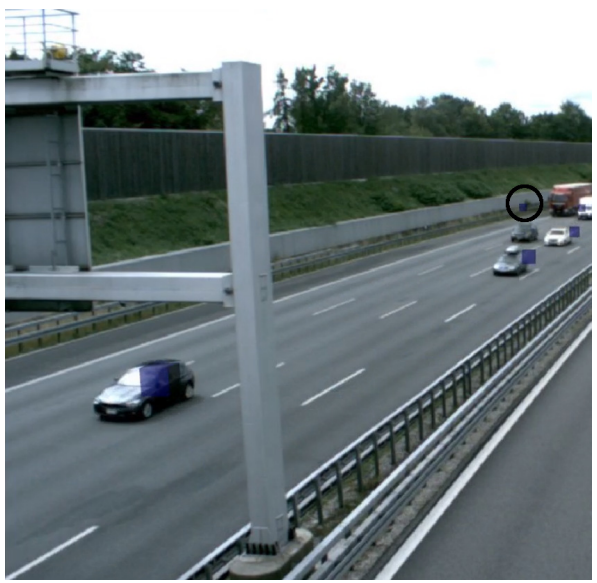

(a)

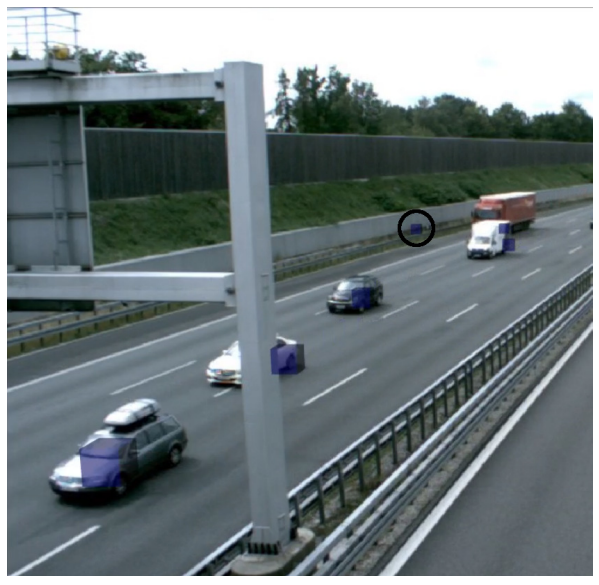

(d)

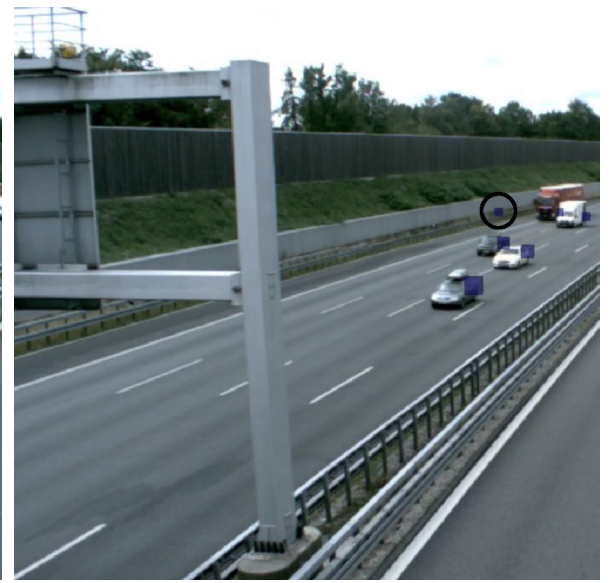

(b)

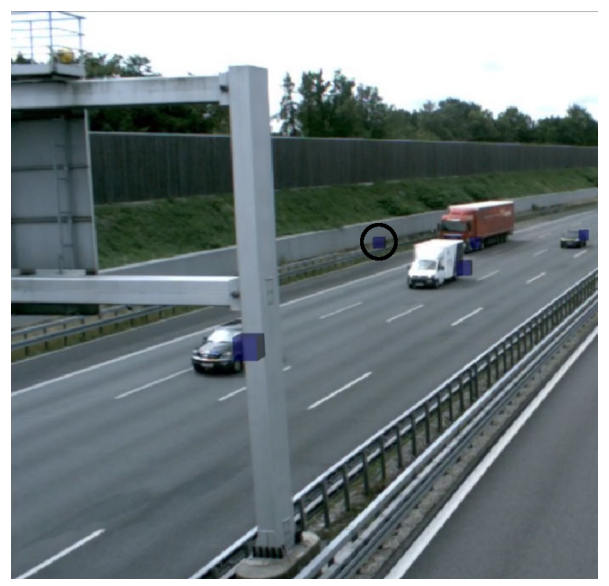

(e)

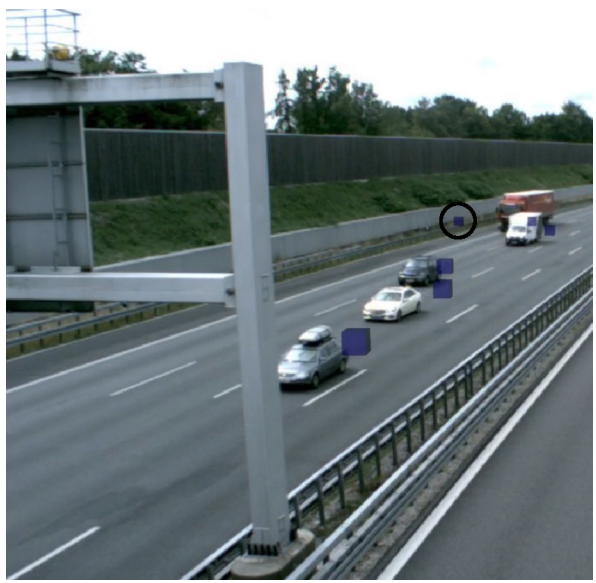

(c)

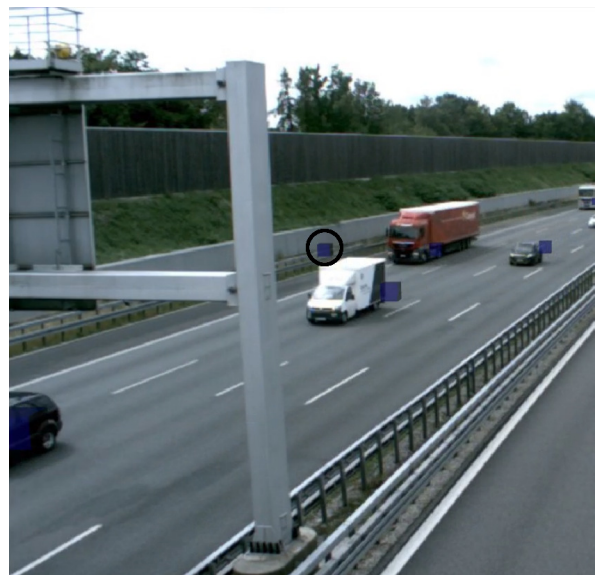

(f)

Fig. 2. Measurements detected by the radar (plotted as cubes) when mapped on the Camera plane highlight the clutter points. The radar could not filter all the possible clutter measurements. In Fig. (a) the clutter point is marked with a circle. Its progression can be seen through all the subsequent pictures (b-f) where it is also encircled.

sensor filtration process have to be addressed in the highlevel fusion process. Therefore, we consider a specific situation (infrastructure radar on a highway) which allows us to make use of higher-level information to get rid of clutter that usual methods cannot deal with.

To understand real clutter phenomenon we analysed the actual radar data which was collected as part of the Providentia Project [24] on German A9 Highway. Figure 2 highlights one set of such data measurements of the radar plotted as cubes on the Camera data. In the Fig. 2(a)-2(f) the clutter point is highlighted with a black circle.

The contribution of this paper is to propose a solution for above kind of realistic clutter scenarios for improved state estimation.

\section{FACTOR GRAPHS}

\section{A. Factor Graphs}

Definition: A factor graph is a bipartite graph $\mathcal{G}_{k}=$ $\left(\mathcal{F}_{k}, \mathcal{V}_{k}, \mathcal{E}_{k}\right)$ with two types of nodes: factor nodes $f_{i} \in \mathcal{F}_{k}$ and variable nodes $v_{j} \in \mathcal{V}_{k}$. Edges $e_{i j} \in E_{k}$ can exist only between factor nodes and variable nodes, and are present if and only if the factor $f_{i}$ involves a variable $v_{j}$ [25].

Factor Graphs can represent Probabilistic Graphical Models (PGM) and can also be used to implement Bayesian networks [26] and Markov Random Fields [27].

We can use the factorized probability distribution to represent the entire trajectories of all the participating vehicles as an optimization problem. For a control input $\mathcal{U}=\left\{u_{i} \mid i \in\right.$ $0, \ldots, n\}$, the localization problem can then be expressed as estimating the trajectory $\mathcal{X}=\left\{x_{i} \mid i \in 0, \ldots, n\right\}$, where $x_{i}$ represents the position in any dimensional coordinates.

$$
P(\mathcal{X}, \mathcal{U}) \propto P\left(x_{0}\right) \prod_{i}^{n} P\left(x_{i+1} \mid x_{i}, u_{i}\right)
$$

For any additional sensor measurements, for example from $\mathcal{S}=\left\{s_{i} \mid i \in 0, \ldots, m\right\}$, the joint density becomes:

$$
P(\mathcal{X}, \mathcal{S}, \mathcal{U}) \propto P\left(x_{0}\right) \prod_{i}^{n} P\left(x_{i+1} \mid x_{i}, u_{i}\right) \prod_{k}^{m} P\left(s_{k} \mid x_{i k}\right)
$$

where $s_{k}$ is the measurement from the sensor $\mathbf{s}$ at position $x_{i k}$. The concept can be extended to any number of sensors. 
Assuming all the distributions as Gaussian, we can convert equation (1) into a least squares formulation as follows:

$$
P\left(x_{i+1} \mid x_{i}, u_{i}\right) \propto \exp \left(-\frac{1}{2}\left\|\mathrm{f}\left(x_{i}, u_{i}\right)-z_{i}^{x}\right\|_{\Sigma_{o}}^{2}\right)
$$

where $x_{i+1}=\mathrm{f}\left(x_{i}, u_{i}\right)+w, \mathrm{f}$ denotes the Odometer function which connects the current position with the previous, $w$ is the unknown Gaussian sensor noise, $z_{i}^{x}$ is the actual Odometer measurement, and $\Sigma_{o}$ is the covariance matrix. For any sensor $s$, the formulation can be written as:

$$
P\left(s_{k} \mid x_{i k}\right) \propto \exp \left(-\frac{1}{2}\left\|\mathrm{~h}\left(x_{i k}\right)-z_{k}^{s}\right\|_{\Sigma_{s}}^{2}\right)
$$

where $s_{k}=\mathrm{h}\left(x_{i k}\right)+v$, h denotes the sensor function to map the current position to the sensor value, $v$ is the unknown Gaussian sensor noise, $z_{k}^{s}$ is the actual sensor measurement with covariance matrices $\Sigma_{s}$.

In this paper, we calculate the maximum likelihood estimation (MLE), $\hat{\mathcal{X}}$, by using the non-linear least square method:

$$
\begin{aligned}
& \hat{\mathcal{X}}=\underset{x}{\arg \max } P(\mathcal{X} \mid \mathcal{S}, \mathcal{U})= \\
& \underset{x}{\arg \min }\left\{\sum_{i=1}^{n}\left\|\mathrm{f}\left(x_{i}, u_{i}\right)-z_{i}^{x}\right\|_{\Sigma_{o}}^{2}+\sum_{k=1}^{m}\left\|\mathrm{~h}\left(x_{i k}\right)-z_{k}^{s}\right\|_{\Sigma_{s}}^{2}\right\}
\end{aligned}
$$

\section{B. Topology Factor}

To perform cooperative localization we had proposed a novel Topology Factor in [14]. Here we give only a brief overview. Topology Factor is formulated using the radar measurements and consists of sum of inter-vehicle distance. The sum of inter-vehicle distance can be written as:

$$
d i s t^{2}=\sum_{i=1}^{N-1} \sum_{j=i+1}^{N}\left(x_{i}^{r}-x_{j}^{r}\right)^{2}
$$

where $x_{i}^{r}$ represents the radar measurement for the $i^{t h}$ target.

The corresponding topology formulation can be written as:

$$
\begin{aligned}
P\left(t_{k} \mid\left(x_{1 k}\right) \cdots\left(x_{N k}\right)\right) & \propto \\
& \quad \exp \left(-\frac{1}{2}\left\|\mathrm{~d}\left(\left(x_{1 k}\right) \cdots\left(x_{N k}\right)\right)-z_{k}^{t}\right\|_{\Sigma_{t}}^{2}\right)
\end{aligned}
$$

where $t_{k}=\mathrm{d}\left(\left(x_{1 k}\right) \cdots\left(x_{N k}\right)\right)-r_{k}$, d denotes the topology function which maps the sensor measurement to the current position, $r_{k}$ is the corresponding Gaussian noise and $z_{k}^{t}$ is the topology measurement calculated from the actual radar measurements with covariance matrices $\Sigma_{t}$.

The Topology measurement is a pseudo measurement, therefore we also need to calculate the covariance, $\Sigma_{t}$, for it. If $\sigma_{x_{i}}^{2}$ is the $x_{i}$ variances for the radar, then we have (see [28] for more details) $\operatorname{Cov}(x)=\operatorname{diag}\left[\sigma_{x_{1}}^{2}, \cdots, \sigma_{x_{n}}^{2}\right]$. Then we obtain the covariance for the topology estimate as:

$$
\sigma_{\text {top }}^{2}=\mathbf{M} \cdot \operatorname{Cov}(x) \cdot \mathbf{M}^{T}
$$

where $\mathbf{M}$ is a $1 \times n$ matrix as follows:

$$
\mathbf{M}=\left[\frac{\partial}{\partial x_{1}}(d), \cdots, \frac{\partial}{\partial x_{n}}(d)\right]
$$

\section{Smoothing}

The formulated factor graph is solved using the Levenberg Marquardt Optimizer. Using an initial estimate $x_{0}$ it iteratively finds an update $\Delta$ from the linearized system:

$$
\underset{\Delta}{\arg \min }\left\{J\left(x_{0}\right) \Delta-b\left(x_{0}\right)\right\}
$$

where $J\left(x_{0}\right)$ is the sparse Jacobian Matrix at the current linearization point $x_{0}$ and $b\left(x_{0}\right)=f\left(x_{0}\right)-z$ is the residual for given the measurement z. After solving (5), the linearization point is updated to the new estimate $x_{0}+\Delta$. Further detail on this process is presented within [29].

\section{Solution}

The Topology Factor requires the coordinates of the targets detected by the radar. The factor performs optimal when the radar detects the same number of targets as the number of pair of Odometer/GPS measurements received from the vehicles. However, in presence of clutter as seen in Section III, radar will result in more measurements than the targets, but the fusion system will still receive same number of Odometer/GPS pair of measurements. This results in more Topology Factors between the states which influence the final state estimation.

For example, for 2 vehicles, an extra clutter measurement means a total of 3 Topology Factors $\left({ }^{3} C_{2}=3\right)$. That is, 2 of the 3 factors contribute false information for the state estimation. The problem becomes worse for higher number of targets or higher number of clutter measurements. For example for 1 clutter measurement for 4 targets results in 5 factors, 4 of which $(80 \%)$ are based on the clutter measurement. And 2 clutter measurements for 2 targets, gives 6 Topology Factors, of which 5 are false.

Sünderhauf et al. [22] proposed the solution of Switchable Constraints. This added a latent variable which "switches off" the factors that contribute higher errors to the final state estimation. A possible solution would be to apply the Switchable Constraints for the Topology Factors, i.e. introduce one switch variable per Topology Factor. The Topology Factors contributing higher error to the final state estimates would be switched off, i.e. the ones from the clutter measurements. Applying Switchable Constraints from [22] on Topology Factor from equation (2) results in :

$$
\begin{aligned}
P\left(t_{k} \mid\left({ }^{N} \mathbb{X}_{n}\right), S_{k j}\right) & \propto \\
& \exp \left(-\frac{1}{2}\left\|\Psi\left(S_{k j}\right) \cdot\left(d_{r}\left({ }^{N} \mathbb{X}_{n}\right)-z_{k j}^{t}\right)\right\|_{\Sigma_{t j}}^{2}\right)
\end{aligned}
$$

where $\Psi$ is the switch function, $S_{k j}$ is the switch variable for the $j^{t h}$ factor. $N$ is the number of radar measurements (including clutter) and $\left({ }^{N} \mathbb{X}_{n}\right)$ is the set of $n$ measurements where $n$ is the number of Odometer/GPS pairs received of $x_{k}$, assuming $N \geq n$.

On the other hand, we know, among all the possible Topology Factors, only one of them is based on the true radar measurement. Therefore, only that should result in the least error. Hence, instead of the Topology Factor controlled by a 
switch variable, we propose a Minimization Topology Factor as follows:

$$
\begin{aligned}
& P\left(t_{k} \mid\left({ }^{N} \mathbb{X}_{n}\right)\right) \propto \\
& \quad \exp \left(-\frac{1}{2}\|\min \|\left(\mathrm{d}\left({ }^{N} \mathbb{X}_{n}\right)-z_{k j}^{t}\right)\|\|_{\Sigma_{t}^{\max }}^{2}\right)
\end{aligned}
$$

where $\min$ is the minimization factor which returns the minimum error from all the possible "sub-factors" from the set of measurements in $\left({ }^{N} \mathbb{X}_{n}\right)$.

Important point which needs to noted here is that for this Minimization Factor we require covariance which is maximum, i.e. $\Sigma_{t}^{\max }$. It is shown in the equations (3) and (4), each factor requires its own derived covariance. The Minimization Topology Factor constitutes of sub-factors therefore it requires a covariance which covers all of the sub-factors. Therefore we choose the biggest covariance from all possible covariances and use that as the covariance for the Minimization Factor. Therefore $\sigma_{\max }^{2}$ for Topology Factor can be written as:

$$
\sigma_{\max }^{2}=\max \left(\mathbf{M} \cdot \operatorname{Cov}(x) \cdot \mathbf{M}^{T}\right), \forall(x) \subset\left({ }^{N} \mathbb{X}_{n}\right)
$$

where $\mathbf{M}$ is a $1 \times n$ matrix as defined in equation (4).

The solution can be understood from the Fig. 3 representing states of two vehicles. Figure 3(a) represents the ideal scenario of no clutter, hence we have only one Topology Factor. Figure 3(b) represents the scenario with one clutter measurement, hence we have three possible Topology Factors. The square in black represents the one generated from the measurement from true targets. Figure 3(c) represents when Switchable Constraints are implemented for the Topology Factor. Finally, Fig. 3(d) represents the case proposed in this paper. The Minimization Topology Factor contains sub-factors for all the possible combinations (here 3 ). And only the one which results in the least error is chosen.

\section{EVALUATion}

\section{A. System Setup}

The proposed solution is evaluated on a simulated data set which is based on real radar observations on German A9 Highway. The simulation is implemented with up to four vehicles on a highway for 200 steps. To implement the factor graphs and the corresponding factors we use the Georgia Tech Smoothing and Mapping (GTSAM) open source library [12].

Simulated vehicles have Odometer sensors to measure their relative movement per unit time. They are also equipped with GPS Sensor to provide the location in global coordinates. A radar sensor is assumed to be located outside the simulated vehicles, and provides location of the observed vehicles within its local coordinate system. No configuration information for the radar is available and hence the transformation between the two coordinate systems, internal to vehicle and external radar, is unknown.

All the sensors are assumed to have zero mean Gaussian noise. The covariances are assumed as $\operatorname{diag}[1.0,1.0] \mathrm{m}$ and $\operatorname{diag}[10.0,10.0] m$ for the Odometer and the GPS respectively.

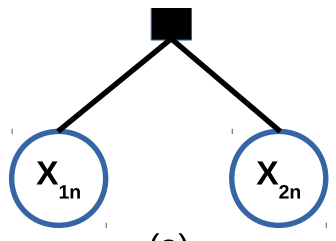

(a)

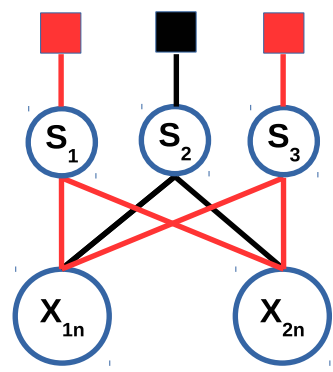

(c)

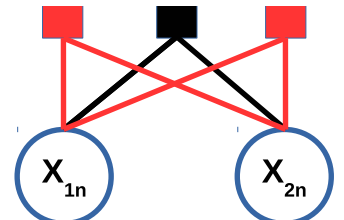

(b)

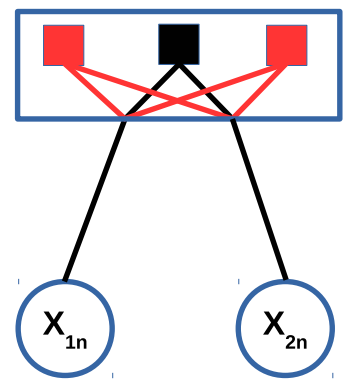

(d)
Fig. 3. State of two vehicles and the Topology Factor. Filled square represents the Topology Factor constructed with radar measurements. Circle with $X_{y z}$ represents the $z^{t h}$ state estimation of $y^{t h}$ target. Circle with $S_{i}$ represent the Switch Variable for the $i^{t h}$ Topology Factor. (a) The ideal case of no clutter, there exists only one Topology Factor for two targets. (b) The radar gives three measurements with one of them being a clutter, hence there are three possible Topology Factors. The black box represents the Topology Factor from true target measurements. (c) Possible solution using Switch Variables for Topology Factor for the scenario depiction in 3(b) (d) Minimization Topology Factor which has three "sub-factors" and uses the one which results in least error for 3(b).

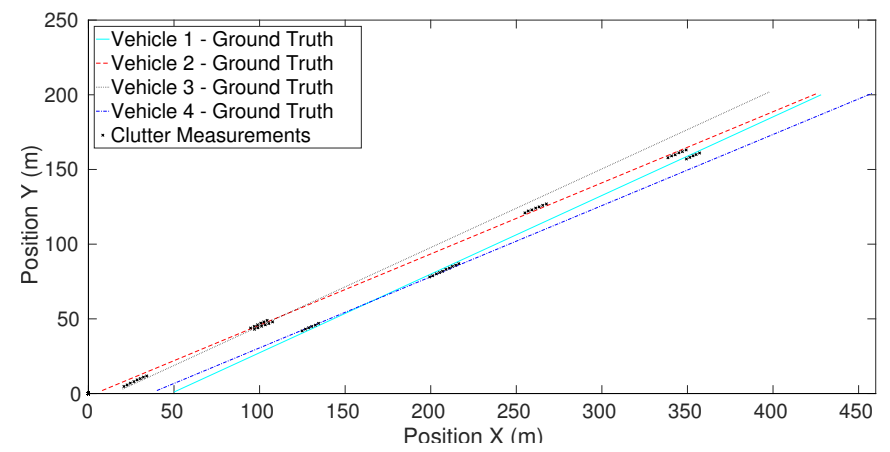

Fig. 4. Ground Truth and upto 3 simultaneous clutter measurements for 4 vehicles on a highway.

We assume the step interval $T$ to be 1 . The radar has one valid reading for the true target with covariance $\operatorname{diag}[0.1,0.1] \mathrm{m}$.

As already mentioned in Section III, MTI Radars are used in Infrastructure and are capable of filtering the static clutter using hardware filters. The big risk of clutter comes from "Ghost Objects" which result from motion originating from phenomena like high winds, rain or reflections. From our observations of the recorded data, we notice the clutter is most likely seen moving parallel to the vehicle. Therefore, clutter measurements are simulated which move parallel to the vehicle movement.

A sample of Ground Truth and corresponding clutter measurements of upto 3 simultaneous clutter measurements for 4 vehicles on a highway is shown in Fig. 4. By "upto 3 si- 
multaneous clutter measurement", we mean that the simulator randomly generates the clutter measurements, therefore it can be only 1 or 2 or 3 measurements.

Various characteristics for the simulated clutter are defined rigorously. The clutter can appear along any randomly chosen vehicle (any of the 4 simulated vehicles). The length of the clutter trajectory is also random with 5 to 10 time steps. The clutter can occur on any side of the simulated vehicle. The clutter measurements are divided into sets of 1 to 5 . Therefore, there are at least 5 clutter measurements in one simulation. The maximum number of simultaneous clutter measurements at any given time step is 3 . So theoretically, the maximum number of clutter points in the system is $3 \times 5 \times 10=150$.

The simulation also makes sure that clutter measurements should not jump around or across the simulated vehicles at every time step. In addition, after one set of clutter is generated, the system waits for at least 30 time steps before a new set may be randomly generated. We randomize the various properties of the clutter measurements in order to get a realistic simulation of the system.

The performance is measured by calculating the RMSE value for the complete system. The total error is the sum of the RMSE of each vehicle for $n$ steps:

$$
\mathrm{RMSE}=\sqrt{\frac{1}{n} \sum_{i=1}^{n}\left(x_{i}^{e s t}-x_{i}^{\text {GroundTruth }}\right)^{2}}
$$

We perform the simulation, and compare and contrast our results five ways. Various cases for the comparison are:

1) the fused trajectory only using Odometer and GPS measurements.

2) the fused trajectory for Odometer, GPS measurements and Topology Factor for each state (assuming no clutter).

3) the fused trajectory for Odometer, GPS measurements and multiple Topology Factors for each state (assuming clutter). This implies we incorporate all the Topology Factors resulting from radar.

4) the fused trajectory for Odometer, GPS measurements and multiple Topology Factors for each state (assuming clutter) with Switchable Constraints. The switch variables control the contribution of each of the Topology Factor. We use the linear version of the switch variable.

5) the fused trajectory for Odometer, GPS measurements and Minimization Topology Factor with all possible Topology Factors as sub-factors for each state (assuming clutter). That is, the solution proposed in this paper.

We perform a Monte Carlo simulation of the above mentioned cases 1000 times and analyse the results.

\section{B. Results}

First consider the simulated scenario highlighted in Fig. (4). For the simulation plotting all the fused trajectories does not highlight the differences between the 5 different cases discussed in the previous "System Setup" section as they are too dense. Therefore, we plot the total system RMSE and analyse how much error did various cases contributed to the

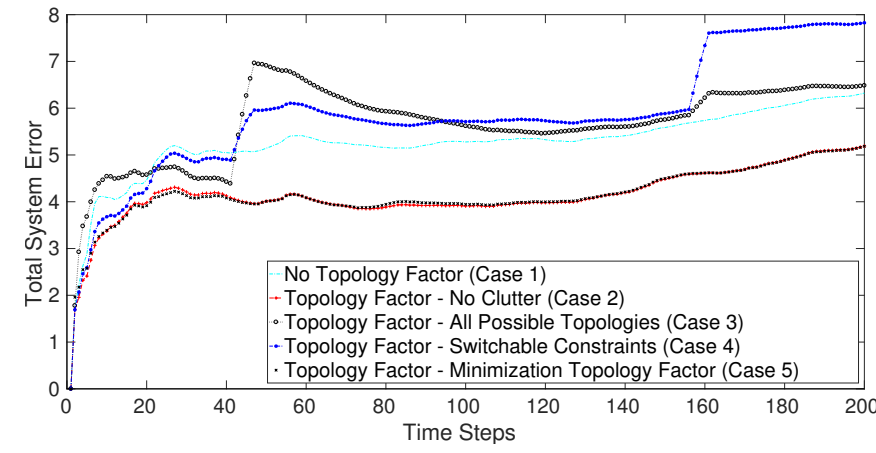

Fig. 5. Total System RMSE for all Cases for the 4 vehicles with clutter for scenario presented in Fig. 4.

system. Figure (5) shows the RMSE of the complete system for the 5 cases for Fig. (4).

It can be seen that for a system without any clutter, the Topology Factor performs better than the one without Topology Factor. The RMSE plotted in the Fig. (4) is for 4 vehicles and 54 clutter measurements. It can be seen that for adding Topology Factor with Switchable Constraints and adding all Topology Factors, both perform worse than the ideal solution of no Clutter. Although it is interesting to see adding all the Topology Factors performs better than when Topology Factors are added with Switchable Constraints. On the other hand, the solution proposed in this paper performs almost the same as the one without clutter. It also converges almost at the same rate. Between time steps 20 and 40 the Minimization Topology Factor seems to perform better than no clutter scenario. This is possible when the randomly simulated clutter measurement is near to the ground truth than the one considered as the true measurement.

It is likely both other solutions, one with Switchable Constraints and using all the Topology Factors) for Fig. (4) will eventually converge but time is a critical resource for Autonomous or Highly Automated Vehicles. Hence overall our solution not only converges faster but also performs as good as the ideal case of no clutter for the given simulated data.

Further analysis and comparison of the results we refer to the Table I, which shows the average RMSE for 1000 iterations for 2,3 and 4 vehicles. The simulation is run for all the Cases and for upto 1,2 and 3 simultaneous clutter measurements. Each row in the table uses the same set of simulated data. This implies that for a simulation run for upto 1 clutter measurement for 2 vehicles all the 5 cases use the same set. Case 1 and 2, ignore the clutter measurements. The RMSE is rounded off to 4 decimal points. The last row mentions the average number of clutter measurements rounded off to an integer.

It can be clearly seen from Table I that among all the cases where clutter measurements are used to construct the Topology Factor, the solution proposed in this paper is superior. The performance does not match the ideal case, but this is expected as sometimes the solution may use a clutter measurement during optimization because the Minimization 
TABLE I

AVERAGE RMSE FOR 1000 ITERATIONS FOR 2, 3 AND 4 VEHICLES.

$(\mathrm{TF}=$ TOPOLOGY FACTOR, ADD ALL $=$ ADD ALL POSSIBLE TOPOLOGY FACTORS,

$\mathrm{SC}=$ TOPOLOGY FACTOR WITH SWITCHABLE CONSTRAINTS, MIN = MINIMIZATION TOPOLOGY FACTOR)

\begin{tabular}{|c|c|c|c|c|c|c|c|c|c|c|c|}
\hline \multirow[b]{2}{*}{ Vehicles } & \multirow[b]{2}{*}{$\begin{array}{c}\text { No TF } \\
\text { (Case 1) }\end{array}$} & \multirow[b]{2}{*}{$\begin{array}{l}\text { TF/No Clutter } \\
\text { (Case 2) }\end{array}$} & \multicolumn{3}{|c|}{ TF/Up-to 1 Clutter } & \multicolumn{3}{|c|}{ TF/Up-to 2 Clutter } & \multicolumn{3}{|c|}{ TF/Up-to 3 Clutter } \\
\hline & & & $\begin{array}{l}\text { Add All } \\
\text { (Case 3) }\end{array}$ & $\begin{array}{c}\text { SC } \\
\text { (Case 4) }\end{array}$ & $\begin{array}{c}\text { Min } \\
\text { (Case 5) }\end{array}$ & $\begin{array}{l}\text { Add All } \\
\text { (Case 3) }\end{array}$ & $\begin{array}{c}\text { SC } \\
\text { (Case 4) }\end{array}$ & $\begin{array}{c}\text { Min } \\
\text { (Case 5) }\end{array}$ & $\begin{array}{l}\text { Add All } \\
\text { (Case 3) }\end{array}$ & $\begin{array}{c}\text { SC } \\
\text { (Case 4) }\end{array}$ & $\begin{array}{c}\text { Min } \\
\text { (Case 5) }\end{array}$ \\
\hline \multirow[t]{3}{*}{2} & 4.2194 & 3.1798 & 7.1168 & 7.1168 & 3.2173 & & & & & & \\
\hline & 4.2114 & 3.1869 & & & & 15.3884 & 15.3884 & 3.2545 & & & \\
\hline & 4.2023 & 3.1837 & & & & & & & 22.2722 & 22.2722 & 3.2871 \\
\hline \multirow[t]{3}{*}{3} & 5.1788 & 4.1613 & 5.6935 & 5.4637 & 5.2990 & & & & & & \\
\hline & 5.1888 & 4.1854 & & & & 6.2963 & 6.2185 & 5.5548 & & & \\
\hline & 5.1753 & 4.1564 & & & & & & & 7.7915 & 6.8170 & 5.7304 \\
\hline \multirow[t]{3}{*}{4} & 5.9432 & 4.7399 & 4.8610 & 5.0605 & 4.8079 & & & & & & \\
\hline & 5.9562 & 4.7497 & & & & 5.1731 & 6.1106 & 4.8836 & & & \\
\hline & 5.9629 & 4.7642 & & & & & & & 5.4044 & 7.0797 & 4.9908 \\
\hline Average & Clutter V & les in System & & 22 & & & 33 & & & 45 & \\
\hline
\end{tabular}

Topology Factor returned a lower error. And since the radar has a higher precision therefore the lower error gets a higher weight and the solution may be swayed away from the true value. Nevertheless, overall the solution successfully stops the effect of the false information and lets the optimizer converge to a solution which near to the ground truth.

For lower number of clutter measurements the solution with Switchable Constraints also performs optimally. This is visible for 3 and 4 vehicles for upto 1 clutter measurement. In this scenario only an average of 22 clutter points were present for 3 and 4 vehicles. However, for 2 vehicle simulation, when the number of clutter points per vehicle increases, the case 3 of adding all the Topology Factors and case 4 for using Switchable Constraints both result in bigger RMSE errors. On the other hand, the proposed solution of case 5 performs optimally. This trend can be seen again in case of upto 3 simultaneous clutter measurements for 2, 3 and 4 vehicles. For upto 3 clutter simultaneous measurements, the simulation generated an average of 45 clutter measurements. Therefore, clutter measurement per vehicle for 2 is quiet high and for per vehicle for 4 vehicles is lower. For 4 vehicles, the Case 3 with Switchable Constraints is able to curtail the influence of Clutter, but our proposed solution is superior and the RMSE is not far from the ideal case of no clutter. And for higher clutter measurements per vehicle, 2 vehicles, the error is very high for both cases 3 and 4 . On the other hand Case 5 still performs optimally.

The RMSE performance for case 3 and 4 , for 2 vehicle scenario is same. This can happen when the switch variables are set to 1 for all the factors thereby all the factors equally contribute to the final optimization. Another interesting observation is, when Case 3 (that is adding all the Topology Factors) performs better than the case 4 (that is the Topology Factor with Switchable Constraints). This is possible when the optimizer ends up tuning the wrong Topology Factor with higher contribution.

Our approach avoids both the pitfalls as the Minimization
Topology Factor partially controls the optimizers behaviour.

The last row of Table I mentions only the average clutter measurements in the system. The actual minimum, maximum and average number of clutter values for 1000 iterations in each case is presented in Table II. As it can be seen, during our simulation there was always some clutter present in the system.

TABLE II

CLUTTER IN THE SYSTEM FOR 1000 ITERATIONS. NOV = NUMBER OF VEHICLES, MIN = MINIMUM NUMBER, MAX $=$ MAXIMUM NUMBER, AVG $=$ AVERAGE NUMBER

\begin{tabular}{ccccccc}
\hline & \multicolumn{2}{c}{ Up-to 1 Clutter } & \multicolumn{2}{c}{ Up-to 2 Clutter } & \multicolumn{2}{c}{ Up-to 3 Clutter } \\
\cline { 2 - 7 } NoV & Min/Max & Avg & Min/Max & Avg & Min/Max & Avg \\
\hline 2 & $5 / 46$ & 22.3 & $5 / 83$ & 33.4 & $5 / 116$ & 44.5 \\
\hline 3 & $5 / 49$ & 22.1 & $5 / 81$ & 33.6 & $5 / 113$ & 45.9 \\
\hline 4 & $5 / 47$ & 22.4 & $5 / 86$ & 33.4 & $5 / 123$ & 44.7 \\
\hline
\end{tabular}

\section{Remarks}

The Minimization Topology Factor proposed in this paper retains all the properties of the original Topology Factor; i.e. bandwidth limitations; data association uncertainties; unknown coordinate transformations; and scalability. Additionally, it can handle clutter scenario and is not dependent on any other external solution.

Current implementation of the Minimization Topology Factor uses the linear search for the minimum error. However, the "sub-factors" can be executed in parallel, thereby avoiding any performance loss.

Presently, the solution is implemented as a batch process. A online solution like iSAM2 of GTSAM can be used for run-time state estimation. Further it also has the potential of supporting plug-and-play paradigm [30].

\section{CONCLUSION}

With Intelligent Highways becoming a reality, cooperative localization is no longer constrained to laboratories. However, 
false positive measurements influence the final state estimates which can be dangerous for the safety critical Autonomous and Highly Automated Vehicles. Therefore there is a need of robust state estimation process which can handle such false positive measurements. The Minimization Topology Factor is an attempt in this direction for Graph Based Solutions which can provide resilience against such clutter, that may have escaped the initial RADAR filtering. Results, from simulation set up built from the observation of real data, highlight that a graph with Minimization Topology Factors is able to successfully avoid the influence of clutter measurements on the final state estimation. Although the RMSE performance does degrade, when compared against the ideal situation of only Topology Factors and no clutter, but the degradation is small and the system performs better than Switchable Constraints. Therefore, this has a potential to solve the challenge of cooperative localization in realistic dynamic scenarios where (1) location and configuration of RADAR is unknown; and (2) clutter, obscuration and miss detections degrade the state estimates.

Future work will focus on the evaluation of the presented approach for vehicle systems with incremental smoothing using real data.

\section{ACKNOWLEDGMENT}

This work was supported by BMWi IKT III SADA Project http://www.projekt-sada.de/ and BMVI Providentia Project http://testfeld-a9.de.

\section{REFERENCES}

[1] A. Osseiran, F. Boccardi, V. Braun, K. Kusume, P. Marsch, M. Maternia O. Queseth, M. Schellmann, H. Schotten, H. Taoka, H. Tullberg, M. A. Uusitalo, B. Timus, and M. Fallgren, "Scenarios for $5 \mathrm{~g}$ mobile and wireless communications: the vision of the metis project," IEEE Communications Magazine, vol. 52, no. 5, pp. 26-35, May 2014.

[2] R. Kurazume, S. Nagata, and S. Hirose, "Cooperative positioning with multiple robots," in Robotics and Automation, 1994. Proceedings., 1994 IEEE International Conference on, May 1994, pp. 1250-1257 vol.2.

[3] S. I. Roumeliotis and G. A. Bekey, "Distributed multirobot localization," IEEE Transactions on Robotics and Automation, vol. 18, no. 5, pp. 781795, Oct 2002.

[4] N. Karam, F. Chausse, R. Aufrere, and R. Chapuis, "Cooperative multivehicle localization," in 2006 IEEE Intelligent Vehicles Symposium. IEEE, 2006, pp. 564-570.

[5] A. Howard, M. J. Matark, and G. S. Sukhatme, "Localization for mobile robot teams using maximum likelihood estimation," in Intelligent Robots and Systems, 2002. IEEE/RSJ International Conference on, vol. 1, 2002, pp. 434-439 vol.1.

[6] A. Ahmad, G. D. Tipaldi, P. Lima, and W. Burgard, "Cooperative robot localization and target tracking based on least squares minimization," in 2013 IEEE International Conference on Robotics and Automation, May 2013, pp. 5696-5701.

[7] D. Fox, W. Burgard, H. Kruppa, and S. Thrun, "A probabilistic approach to collaborative multi-robot localization," Autonomous robots, vol. 8 , no. 3, pp. 325-344, 2000.

[8] H. Li and F. Nashashibi, "Cooperative multi-vehicle localization using split covariance intersection filter," IEEE Intelligent Transportation Systems Magazine, vol. 5, no. 2, pp. 33-44, Summer 2013.

[9] F. Zhang, H. Stähle, G. Chen, C. Buckl, and A. Knoll, "Multiple vehicle cooperative localization under random finite set framework," in 2013 IEEE/RSJ International Conference on Intelligent Robots and Systems, Nov 2013, pp. 1405-1411.

[10] E. W. Kamen, "Multiple target tracking based on symmetric measurement equations," IEEE Transactions on Automatic Control, vol. 37, no. 3, pp. 371-374, Mar 1992.
[11] V. Savic and S. Zazo, "Nonparametric belief propagation based on spanning trees for cooperative localization in wireless sensor networks," in 2010 IEEE 72nd Vehicular Technology Conference - Fall, Sept 2010, pp. 1-5.

[12] "GTSAM, Georgia Tech Smoothing and Mapping," https://collab.cc.gatech.edu/borg/gtsam/.

[13] R. Kümmerle, G. Grisetti, H. Strasdat, K. Konolige, and W. Burgard, "G2o: A general framework for graph optimization," in Robotics and Automation (ICRA), 2011 IEEE International Conference on, May 2011, pp. 3607-3613.

[14] D. Gulati, F. Zhang, D. Clarke, and A. Knoll, "Vehicle infrastructure cooperative localization using factor graphs," in 2016 IEEE Intelligent Vehicles Symposium (IV), June 2016, pp. 1085-1090.

[15] —, "Graph-based cooperative localization using symmetric measurement equations," Sensors, vol. 17(6), no. 1422, 2017. [Online]. Available: http://www.mdpi.com/1424-8220/17/6/1422

[16] M. I. Skolnik, "Radar handbook," 2008.

[17] B. N. Vo and W. K. Ma, "The gaussian mixture probability hypothesis density filter," IEEE Transactions on Signal Processing, vol. 54, no. 11, pp. 4091-4104, Nov 2006.

[18] B. Ngu Vo and B. Tuong Vo, "Multi-Sensor Multi-object Tracking with the Generalized Labeled Multi-Bernoulli Filter," ArXiv e-prints, Feb. 2017.

[19] R. L. Streit and T. E. Luginbuhl, "A probabilistic multi-hypothesis tracking algorithm without enumeration and pruning," in Proceedings of the Sixth Joint Service Data Fusion Symposium, 1993, pp. 1015-1024.

[20] S. H. Rezatofighi, A. Milan, Z. Zhang, Q. Shi, A. Dick, and I. Reid, "Joint probabilistic data association revisited," in 2015 IEEE International Conference on Computer Vision (ICCV), Dec 2015, pp. 30473055.

[21] D. Gulati, F. Zhang, D. Malovetz, D. Clarke, and A. Knoll, "Robust cooperative localization in a dynamic environment using factor graphs and probability data association filter," in Information Fusion (FUSION), 20th International Conference on, 2017, p. Accepted.

[22] N. Sünderhauf and P. Protzel, "Switchable constraints for robust pose graph slam," in 2012 IEEE/RSJ International Conference on Intelligent Robots and Systems, Oct 2012, pp. 1879-1884.

[23] "Radar Tutorials (on 21 Feb 2018)," http://www.radartutorial.eu.

[24] "Digital Test Field (on 21 Feb 2018)," http://testfeld-a9.de.

[25] F. R. Kschischang, B. J. Frey, and H. A. Loeliger, "Factor graphs and the sum-product algorithm," IEEE Transactions on Information Theory, vol. 47, no. 2, pp. 498-519, Feb 2001.

[26] H.-A. Loeliger, "An introduction to factor graphs," Signal Processing Magazine, IEEE, vol. 21, no. 1, pp. 28-41, 2004.

[27] V. Indelman, S. Williams, M. Kaess, and F. Dellaert, "Information fusion in navigation systems via factor graph based incremental smoothing," Robotics and Autonomous Systems, vol. 61, no. 8, pp. 721-738, 2013.

[28] K. O. Arras, "An introduction to error propagation: Derivation, meaning and examples of equation cy = fx cxfx t." ETH-Zürich, Tech. Rep., 1998.

[29] F. Dellaert and M. Kaess, "Square root sam: Simultaneous localization and mapping via square root information smoothing," Int. J. Rob. Res., vol. 25, no. 12, pp. 1181-1203, Dec 2006. [Online]. Available: http://dx.doi.org/10.1177/0278364906072768

[30] H. P. Chiu, X. S. Zhou, L. Carlone, F. Dellaert, S. Samarasekera, and R. Kumar, "Constrained optimal selection for multi-sensor robot navigation using plug-and-play factor graphs," in 2014 IEEE International Conference on Robotics and Automation (ICRA), May 2014, pp. 663670 . 